\title{
Drowsiness detection using Eye-Blink frequency and Yawn count for Driver Alert
}

\author{
Maganti Manasa, Vikas B, K. Subhadra
}

\begin{abstract}
Several reasons can be sighted for the cause of these road accidents. Few of them include lack of sleep, drunk driving, violation of traffic rules, etc. Amongst them, the state of drowsiness and drunk driving alone contributes to $36 \%$ of accidents. Though a number of national schemes and traffic rules have been implemented to avoid these road accidents, it could only bring down the accident rate by $10 \%$. As car accidents are one of the major issues of concern, this paper will be discussing mainly on Drunk driving or drowsiness. In these recent years, various methods have been proposed to implement drowsiness detection based on Hough transforms. Here, in this paper, we have determined a technique to detect drowsiness among car drivers and alert them whenever they tend to sleep. The algorithm is based on eye-blink and yawn frequency. It deals with an eye blink yawn frequency algorithm that uses eye coordinates to keep track of person and determine the open or closed state of the eye and generate an alarm if the driver is drowsy. The yawn count is determined by checking the frequency of yawn count with a minimum threshold value.
\end{abstract}

Keywords : Drowsiness Detection, Eye-blink frequency, Eye-Aspect-ratio, Euclidean distance, PERCLOS

Abbreviations: EAR- Eye Aspect Ratio, PERCLOSPERcentage of eye CLOSure

\section{INTRODUCTION}

In the contemporary world, the major cause of death is prone to road accidents. They have become very common that approximately 1.35 million people alone die in a year due to these car crashes. The damage is not only caused to the property but also the people traveling inside it. This has caused a significant loss to both the country's GDP and the welfare of the nation. Currently, the transport systems are the quintessential part of human activities. Every traffic management system must take certain measures to avoid these road accidents. Amongst all, fatigue driving can be an important cause for road accidents. Novel methods are introduced to determined and implemented to monitor the level of attention of the driver. Drivers who are in drowsy state usually tend to sleep and might not be able to focus on driving. This happens especially during the early mornings and at late nights while getting back from work. The drowsy state of a person can be easily identified using two parameters which are eye-blink frequency and yawn

Revised Manuscript Received on December 13, 2019.

* Correspondence Author

M.Manasa*, UG student , department of CSE, GITAM University, Visakhapatnam, AP

Vikas B , Assistant Professor, GITAM University, Visakhapatnam, AP

Dr K. Subhadra , Assistant Professor, GITAM University,

Visakhapatnam, AP count. W. Tipprasert, et al. [1] discusses about method of driver eyes closure and yawning detection for drowsiness using Infrared camera. The eye detection process is examined using the signal processing technique. Initially the face is divided into two parts i.e., upper half and lower half. The upper half is used for eye detection which is particularly carried out using the PERCLOS method. The yawn detection is carried out by Histogram of Oriented (HOG) and calculate the scale and vector of each pixel. S. D. Lin et al. [2] presents the statistics of the car accidents that happen frequently due to drowsiness of the car driver. This method basically follows four main steps. They are image pre-processing, face detection, eye state detection and recognition of drowsiness state. Tianyi Hong et al. [3] talks about implementation of drowsiness detection in embedded systems. The detection is done based on few image processing techniques. The object tracking method keeps track of eyes and the driver detection is identified using the PERCLOS method. The binary value of image is determined and when it is less than a certain threshold value, an alarm is sounded. In Pooneh Tabrizi et al. [4] a novel method is proposed where the drowsiness analysis is done using video capturing with a specific algorithm. The algorithm proceeds in two phases where the first state includes variance projection of the curve of eye area and the measure of the distance between iris radius and the two lids. This is a non-intrusive, simple method which did not require any training of the data. Also, drowsiness detection was performed based on the EEG-Based Power Spectrum Analysis [5]. All the electronic devices must be turned off to remove the noise from the environment. The EEG experiments are carried out and change in alpha power spectrum determines the drowsy state.

\section{DEPENDENCIES USED IN THE ALGORITHM}

\section{OpenCV (Opensource Computer Vision)}

This library [6] consists of set of programming functions which provide the functionalities based on computer vision. OpenCV can be used in extension with programming languages like $\mathrm{C} / \mathrm{C}++$ as well. Various tasks involved in Computer Vision such as reading the image, writing it to file or program, displaying images on the screen, change the color space of image, resizing, rotating the image, thresholding, segmentation, edge detection, filtering the image, contouring the images, and many more can be achieved through this library. Along

with these different functionalities, it also extends its support to varied programming languages such ac Python, $\mathrm{C}, \mathrm{C}++$ and also deep learning frameworks like TensorFlow, PyTorch, Caffe. 


\section{Imutils}

This library is mainly used for performing translations, rotation, resizing, skeletonization, and display the images easily using OpenCV and matplotlib libraries. In this paper, it is used to import the coordinates of the face landmarks which are predefined in a .dat file.

\section{Dlib}

Dlib is a machine learning library which is importantly used in frame detection and facial landmark detection. It allows the tracking of multiple objects in a single frame which can be eventually used in object detection. The algorithms used to perform in Bayesian network and Kernel based methods are also depended on this library. This library is a toolkit which is based in linear algebra. It also supports $\mathrm{C}++$ along apart from Python.

\section{METHODOLOGY}

The implementation of drowsiness detection system can be primarily carried out by two methods. The frequency of number of eye blinks and the yawn count. Based on these two parameters, a person can be identified whether or not he is drowsy. Whenever he is drowsy, he tends to sleep more i.e., the frequency of eye closure increases. If this value exceeds the threshold value then an alarm must be generated. Also, the yawn count increases for a certain period of time where both the lips stay apart for few milliseconds. Based on this idea, an implementation is performed using certain dependencies which has led to better performance of the algorithm in terms of speed and accuracy.

Initially, the dependencies or the required libraries for the code are loaded which help in various functionalities while executing the algorithm. This computer vision-based system checks for drowsiness detection of the driver by tracking his face in real-time. An alarm is generated if he is found drowsy. A facial landmarks file is imported into this algorithm which is used to locate the coordinates of the facial features for an individual. These coordinates are useful while drawing the contours around the features and keeping track of the eyes and mouth distance ratio. The pattern and edge detection are implemented using the functions from Imutils library.

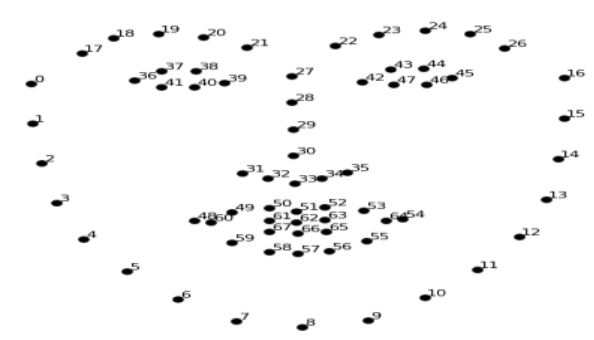

Fig.1 Facial landmarks file used for coordinates

The entire frame is then converted into a grayscale image to determine the subject from the frame. The OpenCV library helps in detecting the subject from the frame. The coordinates which are taken from the dat file are now used to locate the features in the frame.

To determine the contours of eyes, the eye aspect ratio is taken so as to calculate the distance from left edge to right edge of eye. A specific Euclidean function can be used here to calculate the distance between two eyes. Similarly, the mouth distance is also calculated for estimating the frequency of the yawn count.

$$
\mathrm{EAR}=\frac{\left\|p_{2}-p_{6}\right\|+\left\|p_{3}-p_{5}\right\|}{2\left\|p_{1}-p_{4}\right\|}
$$

Whenever the person gets drowsy, the distance between both the eyelids is reduced and the distance between both the lips increases when the person yawns. A fixed threshold value is set for both eye and yawn frequency. If either of those values cross these threshold values, then an alarm is generated on the screen. For 20 consecutive frames, if the eye aspect ratio is found to be less than 0.25 , an alert will be displayed on the frame.

\section{Performance Measures of the Algorithm}

The performance measures [7] of the algorithm used in this method can be calculated using some metrics. The algorithm used in this paper is compared with different techniques which were already implemented [12]. Accuracy, precision, sensitivity and specificity are calculated for 100 sample observations.

\section{Sensitivity:}

The true positive rate for the given sample can be calculated using the sensitivity. In other words, it is the true positive rate. Sensitivity $=\frac{\text { frequency of true positives }}{\text { frequency of true positives }+ \text { frequency of false negatives }}$

\section{Specificity:}

The number of negative observations divided by total number of negatives. It is also known as the true negative rate.

Specificity $=\frac{\text { frequency of true negatives }}{\text { frequency of false positives }+ \text { frequency of true negatives }}$

\section{Precision:}

It is the number of correct positive predictions in the data divided by that of the total number of positive predictions.

Precision $=\frac{\text { frequency of true positives }}{\text { frequency of true positives }+ \text { frequency of false positives }}$

\section{Accuracy:}

The total number of all correct predictions in the set of data by total number of values.

\section{Accuracy $=\frac{\text { frequency of true positives }+ \text { frequency of true negatives }}{\text { frequency of true positives }+ \text { false negatives }+ \text { false positives }+ \text { true negatives }}$}

\section{Recall:}

Recall is measure of true positive values against all positive results calculated.

$$
\text { Recall }=\frac{t p}{t p+f n}
$$




\section{Proposed Algorithm}

It has been seen that most of the algorithms which implement drowsiness detection, Hough transform technique is used on Percentage of Eye Closure (PERCLOS) method. However, it is observed that the novel approach used in this algorithm provides more accurate results over these conventional methods. The algorithm takes input from the video frame and it is processed to find out if the person is drowsy or not. Based on two parameters, i.e., eye closure and yawn frequency, the algorithm rings an alarm.

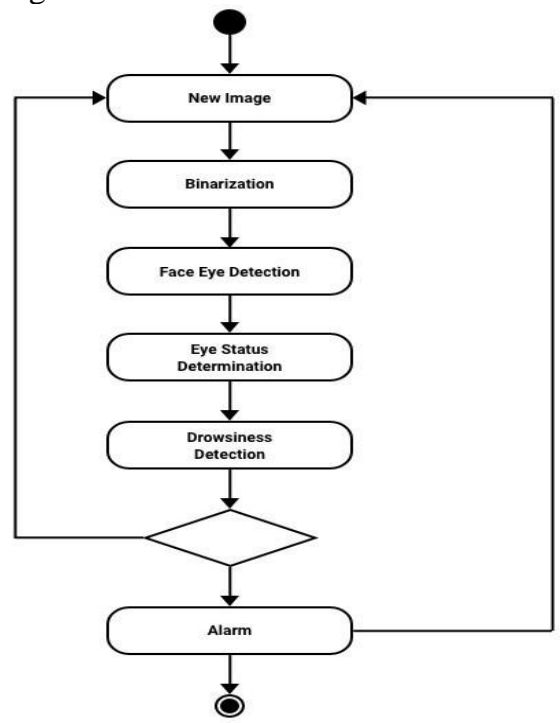

Fig.2 Flowchart of the proposed algorithm

\section{RESUlTs}

The results show various confusion matrix parameters for the given algorithm. Around 100 test samples were taken for testing the performance metrics. The accuracy proved to be $96 \%$ which is more than most of the other algorithms used for drowsiness detection. Although algorithm misses out on certain inputs, it still is able to clearly distinguish the drowsiness and yawn for a frame image.

Table-1 Confusion Matrix Report

\begin{tabular}{|l|l|l|}
\hline & Predicted: Yes & Predicted: No \\
\hline Actual: Yes & 60 & 2 \\
\hline Actual: No & 2 & 36 \\
\hline
\end{tabular}

Table-2 Proposed algorithm report

\begin{tabular}{|l|l|l|l|l|}
\hline & Precision & Recall & F1-score & Support \\
\hline 0 & 0.97 & 0.97 & 0.97 & 62 \\
\hline 1 & 0.95 & 0.95 & 0.95 & 38 \\
\hline Macro average & 0.96 & 0.96 & 0.96 & 100 \\
\hline Weighted Avg & 0.96 & 0.96 & 0.96 & 100 \\
\hline
\end{tabular}

Below are the output screens after the program is run. The algorithm correctly classifies the yawn and eye blink and alerts the driver.

When the eye aspect ratio is less than the set threshold value, then an alarm alerts the driver stating he is drowsy.

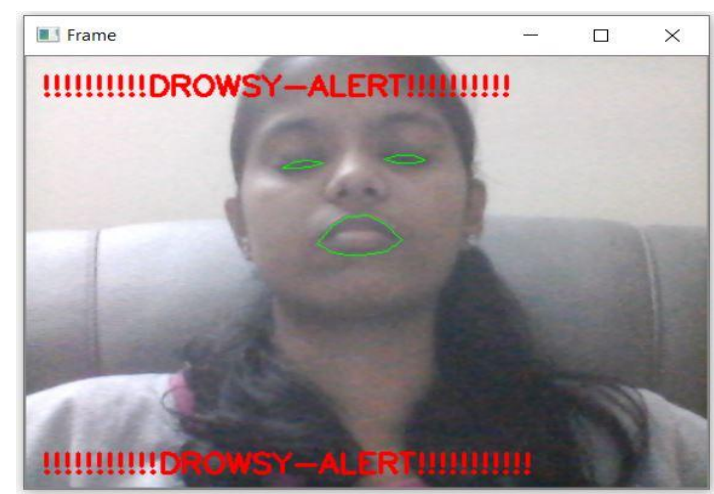

Fig.3 Drowsy alert when the person EAR is less than threshold value

However, if the eyes are opened and the yawn count is greater

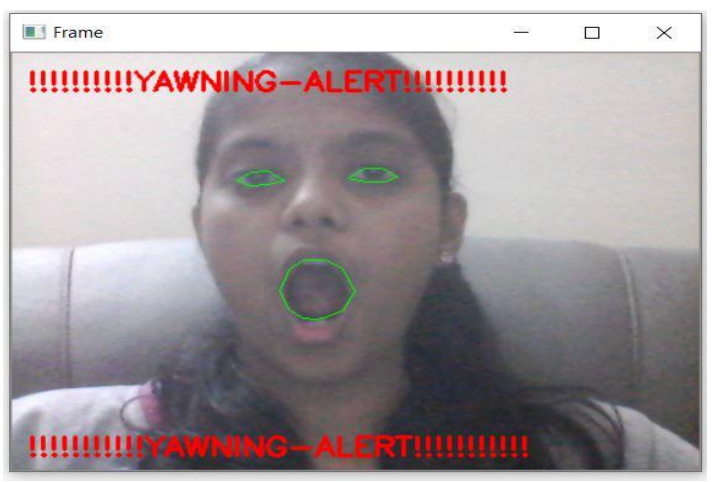

than certain threshold value, the Yawn alert is generated.

Fig.4 Yawn alert generated while coordinates of mouth are distant

Table-3 Accuracy for different methods

\begin{tabular}{|l|l|l|l|l|}
\hline & $\begin{array}{l}\text { Embedded } \\
\text { System }\end{array}$ & $\begin{array}{l}\text { Hough } \\
\text { Transform }\end{array}$ & $\begin{array}{l}\text { Eye State } \\
\text { Compone } \\
\text { nt }\end{array}$ & Our model \\
\hline Accuracy (\%) & $\mathbf{8 9 . 3}$ & $\mathbf{8 7 . 5}$ & $\mathbf{9 4 . 2}$ & $\mathbf{9 6}$ \\
\hline
\end{tabular}

The accuracy is compared between [3], [4], [11] and the model proposed. It is observed that the current model used has the highest accuracy. It is also more efficient as it performs the detection within less time complexity.

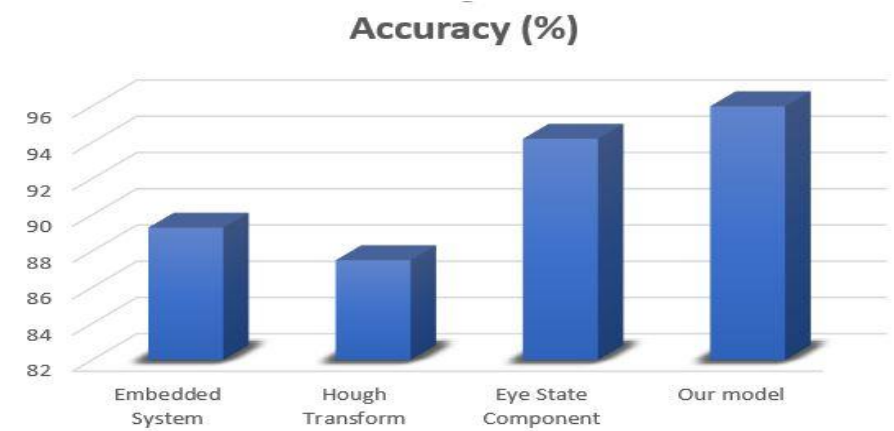

Fig.6 Table comprising of accuracy for different methods 


\section{CONCLUSION}

It is clearly implied from the above results that the accuracy varies from 85-94.5 using other techniques whereas it varies from $91-96 \%$ in our proposed model. It also consumes less time and with minimal code. This model is reliable and can be implemented practically. In the future work, this algorithm can be used as a base and can be implemented in an Android application through API calls. This application can be implemented in real-time to avoid accidents caused by driver's drowsiness.

\section{REFERENCES}

1. W. Tipprasert, T. Charoenpong, C. Chianrabutra and C. Sukjamsri, "A Method of Driver's Eyes Closure and Yawning Detection for Drowsiness Analysis by Infrared Camera," 2019 First International Symposium on Instrumentation, Control, Artificial Intelligence, and Robotics (ICA-SYMP), Bangkok, Thailand, 2019, pp. 61-64

2. S. D. Lin, J. Lin and C. Chung, "Sleepy Eye's Recognition for Drowsiness Detection," 2013 International Symposium on Biometrics and Security Technologies, Chengdu, 2013, pp. 176-179.

3. Tianyi Hong and Huabiao Qin, "Drivers drowsiness detection in embedded system," 2007 IEEE International Conference on Vehicular Electronics and Safety, Beijing, 2007, pp. 1-5

4. P. R. Tabrizi and R. A. Zoroofi, "Drowsiness Detection Based on Brightness and Numeral Features of Eye Image," 2009 Fifth International Conference on Intelligent Information Hiding and Multimedia Signal Processing, Kyoto, 2009, pp. 1310-1313

5. D. Kim, H. Han, S. Cho and U. Chong, "Detection of drowsiness with eyes open using EEG-based power spectrum analysis," 2012 7th International Forum on Strategic Technology (IFOST), Tomsk, 2012, pp. 1-4

6. I. Culjak, D. Abram, T. Pribanic, H. Dzapo and M. Cifrek, "A brief introduction to OpenCV," 2012 Proceedings of the 35th International Convention MIPRO, Opatija, 2012, pp. 1725-1730

7. Vikas B, B. S. Anuhya, Manaswini Chilla and Sipra Sarangi, "A Critical Study of Polycystic Ovarian Syndrome (PCOS) Classification Techniques" IJCEM International Journal of Computational Engineering \& Management, 2018, 2230-7893

8. B. N. Manu, "Facial features monitoring for real time drowsiness detection," 2016 12th International Conference on Innovations in Information Technology (IIT), Al-Ain, 2016, pp. 1-4

9. Y. Katyal, S. Alur and S. Dwivedi, "Safe driving by detecting lane discipline and driver drowsiness," 2014 IEEE International Conference on Advanced Communications, Control and Computing Technologies, Ramanathapuram, 2014, pp. 1008-1012

10. D. Kurian, J. J. P.L., K. Radhakrishnan and A. A. Balakrishnan, "Drowsiness Detection Using Photoplethysmography Signal," 2014 Fourth International Conference on Advances in Computing and Communications, Cochin, 2014, pp. 73-76

11. M. S. Devi, M. V. Choudhari and P. Bajaj, "Driver Drowsiness Detection Using Skin Color Algorithm and Circular Hough Transform," 2011 Fourth International Conference on Emerging Trends in Engineering \& Technology, Port Louis, 2011, pp. 129-134

12. K. Santosh Bhargav, T.Divya Kumari, Dola Sai Siva Bhaskar Thota, Vikas B., "Application of Machine Learning Classification Algorithms on Hepatitis Dataset"International Journal of Applied Engineering Research ISSN 0973-4562, pp. 12732-12737

\section{AUTHORS PROFILE}

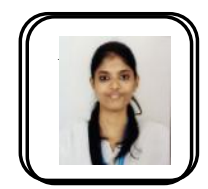

M.Manasa is a student currently pursuing final year in B.Tech, Computer Science \& Engg. from GITAM, Visakhapatnam. Her research interests are in the field of Machine Learning, and Data Science.

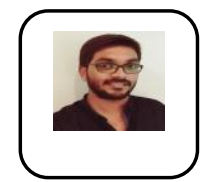

Vikas B is currently working as Assistant Professor in CSE in GITAM. He is member of IAENG and IEEE-CS and his research interests include Data mining, Bioinformatics, Information Security, and Data Sciences.

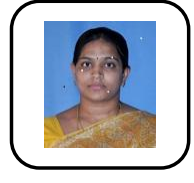

Dr K. Subhadra is currently working as Assistant Professor in CSE in GITAM. She is actively engaged in research in the area of Big Data Analytics and Algorithms. She has a teaching experience of about 13 years 